\title{
STEP Compliant CAD/CAM - Challenges and the Future
}

\author{
Jabran Kayani* $^{*}$ and Pedro Rico
}

School of AerospaceEngineeringRenewable Energy MSc, Kingston University

\begin{abstract}
The quest to increase productivity and profitability in a highly competitive designing and manufacturing industry has been of paramount importance. Improving quality and reliability has been in the fore front of the research to adopt a paradigm shift from ISO 6983 to STEP and its manufacturing off-shoot STEP-NC (ISO 14649). This paper attempts to describe the difference between the prevalent G-Code standard and the emerging STEP-NC standard. The working and methodology of STEP-NC is described and the research being carried out around the globe is mentioned. Lastly the advantages and disadvantages of adopting the new system are discussed.
\end{abstract}

Keywords: CAD, CAM, CNC, STEP-NC; ISO 14649.

\section{INTRODUCTION}

The advent of modern technology has heralded a significant change in human society both as consumers and manufacturers. Manufacturing has come a long way, from manual labour to state of the art multi axis CNC machines and modern production facilities, to compete in the global market and meet the insatiable demands of a highly competitive market. Such competition has enabled new and efficient methods to increase profitability, quality and reliability. These advancements have in turn given rise to a set of inherent problems making manufacturing much more difficult [1]. The last 60 years have seen the evolution of $\mathrm{NC}$ machines to modern multi-axis CNC machines. Despite the change, the G-Code, an international standard ISO 6983 (also known as RS274D) remains the basic programming language [2], which is not adequate for complex geometries and high level machining as it only offers relatively simple machine tool paths and functionality and provides no knowledge of the geometry of the part being machined. The limited ability of the program to be altered on the shop floor and the inability of feed-back data from the workshop to design room renders ISO 6983 inefficient. Fig. (1) briefly shows the process parameters limited by machine axis codes of ISO 6983 [3].

To increase productivity and profitability in this globalized and competitive world, centres of manufacturing are set up near the markets with access to cheap labour, which limits and in many cases hinders the optimal information flow among different production site due to their machine specific nature. A new system of standardized data model is devised to enable optimum flow of information among different centres of production using CNC, namely ISO 14649 (STEP$\mathrm{NC)}[4]$.

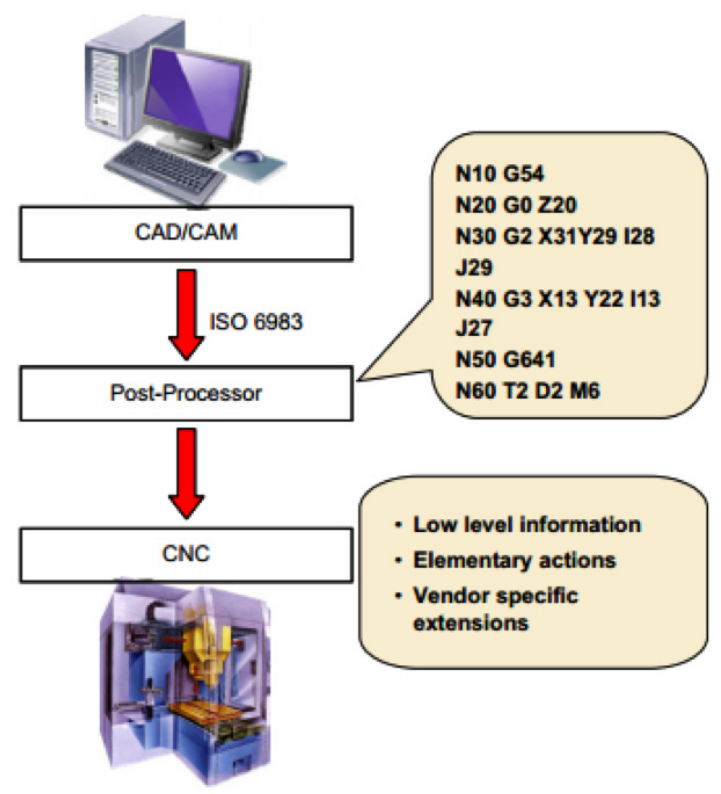

Fig. (1). G-Code programming from (Rauch, et al., 2012).

STEP-NC, unlike the G-code, offers machining for complex geometries and high level task. STEP-NC file is independent of machine specific instructions and tool. STEP-NC file offers an array of useful data such as the features, tools and machining information in compartmentalized "Working Steps" in a "Work Plan", as shown in Fig. (2). The purpose of this standard is to allow the STEP-NC controller to modify and alter these working plans by itself so it can be used on any CNC machine irrespective of the geographical location enabling easy dissemination of data among different manufacturing sites thus increasing profitability [2].

There has been extensive work done on the development of STEP ("Standard for the Exchange of Product model data") ISO 10303 globally to standardise and define the product life cycle and as an apparatus for data exchange amongst different machine systems, CADCAM systems and industries. STEP has various parts such as Application Pro- 


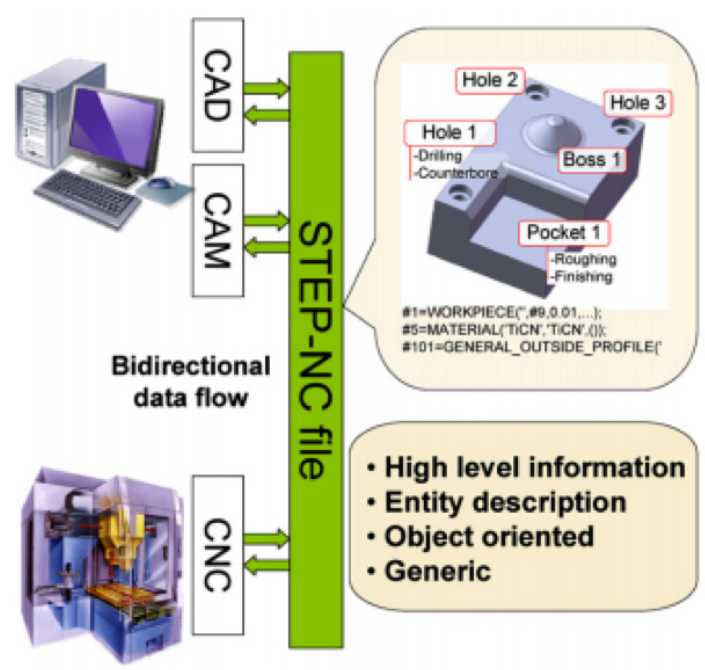

Fig. (2). STEP-NC Programming from (Rauch, et al., 2012).

tocols (APs), Implementation Methods, Description Methods, Information Model, conformance testing framework assembled into Top parts, Environment and Integrated data models. Application Protocols or APs defines the construction and tolerance of a model in various conformance modules [2]. Such as,

- AP-203 - Configuration controlled 3D design,

- AP 210 -Electronic assembly and packaging design,

- AP-224 - Mechanical product definition for process plans using machining features,

- AP-239 - Product life cycle support.

- AP-219 - Dimensional inspection information exchange.

Such a division insures that complete product data including geometry, conformance, tolerances, maintenance, mechanicalproperties and retirement time of the product are transferred between designers and manufacturers irrespective of the different $\mathrm{CNC}$ and design systems being used in the industry to ensure accurate, reliable and quality products [5].

STEP NC (ISO 14649) is an extension of this effort towards a machine model. STEP-NC is designed to be resource independent as it contains extensive information about the "Working Steps", tools, machining operations, tolerances, feed rates and sensor data to provide feedback so that optimal tool path is calculated based on the feedback and the library of information in STEP-NC. Step-NC is intended to be a substitute to RS274D (G-code) as a means to transfer data from a CAD file to a CNC machine because of the inherent object-oriented properties and data models, STEP-NC, compensates the limitations of G-Code, as an axis-based program, to do feature based machining [6].

The purpose of this paper is to demonstrate the ability of ISO 14649 in a globalized manufacturing environment and to compensate and enhance the shortcomings of the still prevalent ISO 6983. This paper also discusses the challenges ISO 14649 faces by the CAx vendors and manufactures in adapting to the new set of programming skills.

\section{LITERATURE REVIEW}

\subsection{STEP NC Data Structure}

EXPRESS language (10303-11: 1994) is the formal language used by STEP and STEP-NC, which insures uniformity, reliability and allows seamless data exchange between CAD, CAPP and CAM. The rules for describing data in a character based physical file (ISO 10303-21: 1994) for EXPRESS/STEP are contained in STEP part 21.[7]

The simplified Fig. (3) shows the STEP-NC data model depicting the three sub-sections of the DATA section, containing a workplan, geometry information and project entity [8].

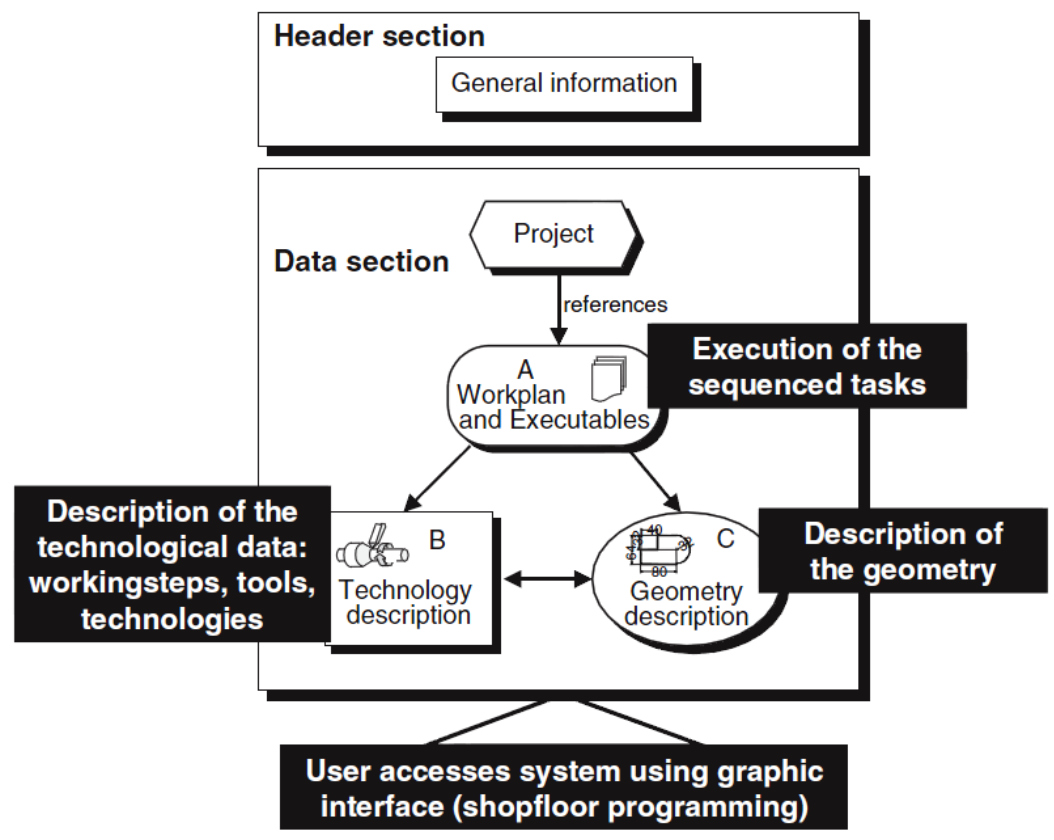

Fig. (3). STEP-NC data structure (Xu \& X.W., 2006). 


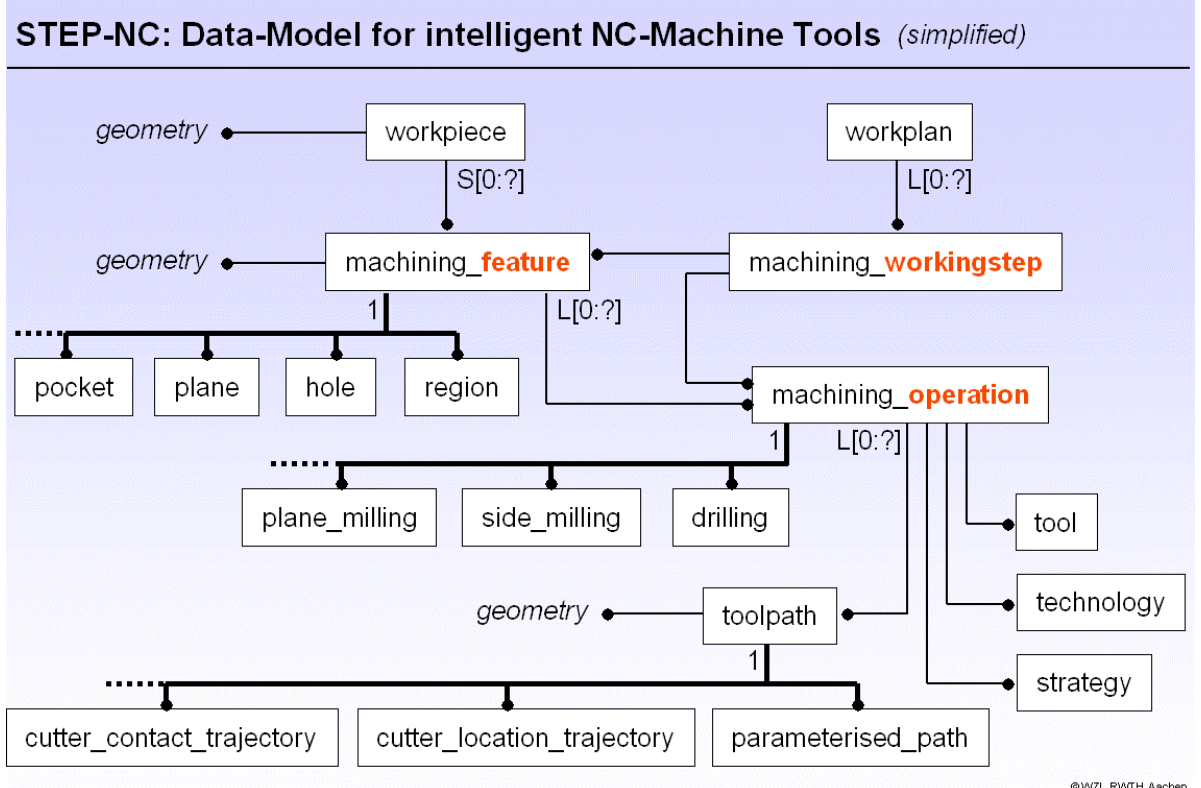

Fig. (4). ISO 14649 "Geometry, technology and process information" from (Xu \& Liu, 2012).

Table 1. Comparison of Part 21 and Part 28 from (XU, et al., 2005).

\begin{tabular}{|c|c|c|}
\hline Comparison Criteria & STEP Part 21 & Early Binding Approach of STEP Part 28 \\
\hline \hline EXPRESS Instances & Yes & Yes \\
\hline Extensibilities & Weak & More \\
\hline Space requirement & Less & Hierarchy structured \\
\hline Structure & Cross Linked base & Yes \\
\hline Data repetition & No & Completely \\
\hline Human interpretable & Partly & Easy \\
\hline Interpretation & Hard & Any part in its hierarchy structure \\
\hline Exchange over the Internet & Via an Individual file & \\
\hline
\end{tabular}

The Workplan contains the commands for manufacturing sequences. These commands further contain the program structures, Numerical Control functions and Working Steps. A portion of the internal structure, as depicted in Fig. (4), shows how the machining tasks and the manufacturing feature are connected by the components in a STEP-NC file [7].

The programming language used by STEP has two formats of operation, namely Part 21 and part 28 for defining STEP data. Part 21 represents STEP objects adequately in text and uses the minimalistic method of writing to make the file size small and prevent overwriting of similar information to avoid errors. Part 28 uses XML which is flexible and upgradable and provides opportunities which lack in part 21. XML has enabled the sharing and editing of STEP entities because of its wide spread use in web browsers. Table 1 compares the Part 21 and 28 file format [2].

\subsection{International Researches into STEP-NC}

There have been major global initiatives for the replacement of the G-Code in favour of ISO 14649 STEP-NC, a robust standard and interoperable system, ranging from the efforts of governments to multinational corporations and organizations.

\subsubsection{Super Model Project}

The purpose of this project was to create a database, employing XML as a medium to store all the necessary information to make a part. Use of XML provided an easy relation between the production plans and the machining steps in the database [2].

\subsubsection{SMS Project}

SMS Project, also known as STEPManufacturing suite. With the collaboration of many countries (Germany, Canada, U.K., U.S.A, Japan and Sweden) and companies (Boeing, STEP Tools Inc., GDLS, SCRA) that took part as a STEP community in the ISO TC 184 SC 4 working groups, helped to derive the implementation architecture and application protocols for STEP enabled part production for the use of commercial and defence industry [2]. 


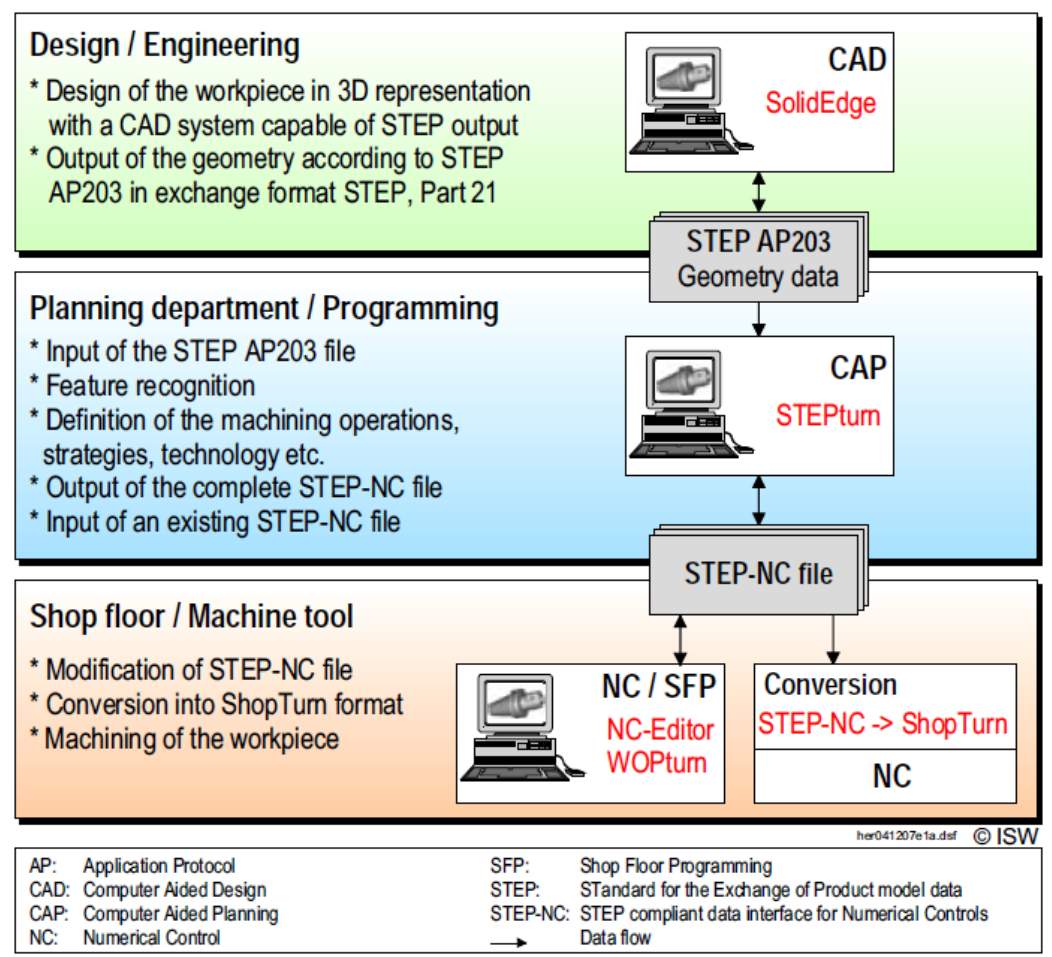

Fig. (5). Turning process chain from (Yusof, 2009).

\subsubsection{RAMP Project}

The purpose of the RAMP "Rapid Acquisition of Manufactured Parts" Project was to create application standards for electrical and mechanical manufacturing. This project also achieved success by the development and application of AP 224 [2].

\subsubsection{Intelligent Manufacture for STEP-NC-Compliant Machining and Inspection}

EPSRC in 2004 worked on Intelligent manufacture for STEP-NC-compliant machining and inspection by extending the work on an EU research project to take measurements while the product is being machined and to research the new STEP-NC standards to be applied to a CMM (Co-ordinate Measuring Machine) and a CNC machine [2].

While manufacturing excessive machine forces, tool wear, chatter and over-loading hasa detrimental effect on the productivity, time, machine performance and part quality. Inspection of the finished part to maintain the product quality is a reactive approach to maintaining the machine performance and product quality. G-Code's inherent deficiencies in defining the tool characteristic, part information and optimization controls renders G-Code highly inefficient in performing the real-time optimization and inspection needed for modern day machining processes. Controlling and monitoring machining parameters during the machining process and observing the tool behaviour allows dynamic quality control and corrective measures to be taken appropriatelyin real-time. STEP-NC high-level object-oriented working environment provides the perfect prospect to readjust for the dynamic, dimensional, static and surface roughness errors through the process control feedback [7].

\subsection{STEPTurn}

Developed by the researchers of the University of Stuttgart and Siemens, a CNC vendor, STEPTurn is CAPP system linking CAD and CAM to implement STEP and STEPNC standards for turned parts. Fig. (5) showsthe geometry information about the work piece being read from STEP AP203 file by STEPTurn which then defines the machining operations and strategies and outputs a STEP-NC file. In experiments, relative simple working steps have been used to machine cones and cylinders on a 2-axis machine which doesn't properlyreflecttheproficiency of the system [9].

\subsection{6. $A B-C A M$}

Agent based system which was developed in the UK is a STEP-complaint system with an NC data support structure. Fig. (6) shows how the AB-CAM system uses STEP data to translate the built in geometric and manufacturing procedures using feature information and generating machine specific part programs and process plans. The result of the ABCAM system is a STEP-compliant file which links CAD and CAM systems through a process plan. This process plan produces a WorkingStep for a STEP-NC file by generating cutting operations, process type features and clamping operations [10].

The purpose of the above mentioned researches and many other un-mentioned researches in the field of STEP and STEP-NC is to achieve unhindered product information and data exchange and flow by using STEP-NC and STEP as a co-ordinated format for NC Manufacturing and design process respectively. Application Protocol (AP) has provided an optimal environment for the integration of STEP and STEP-NC. An AP 203 file contains a design model for a 


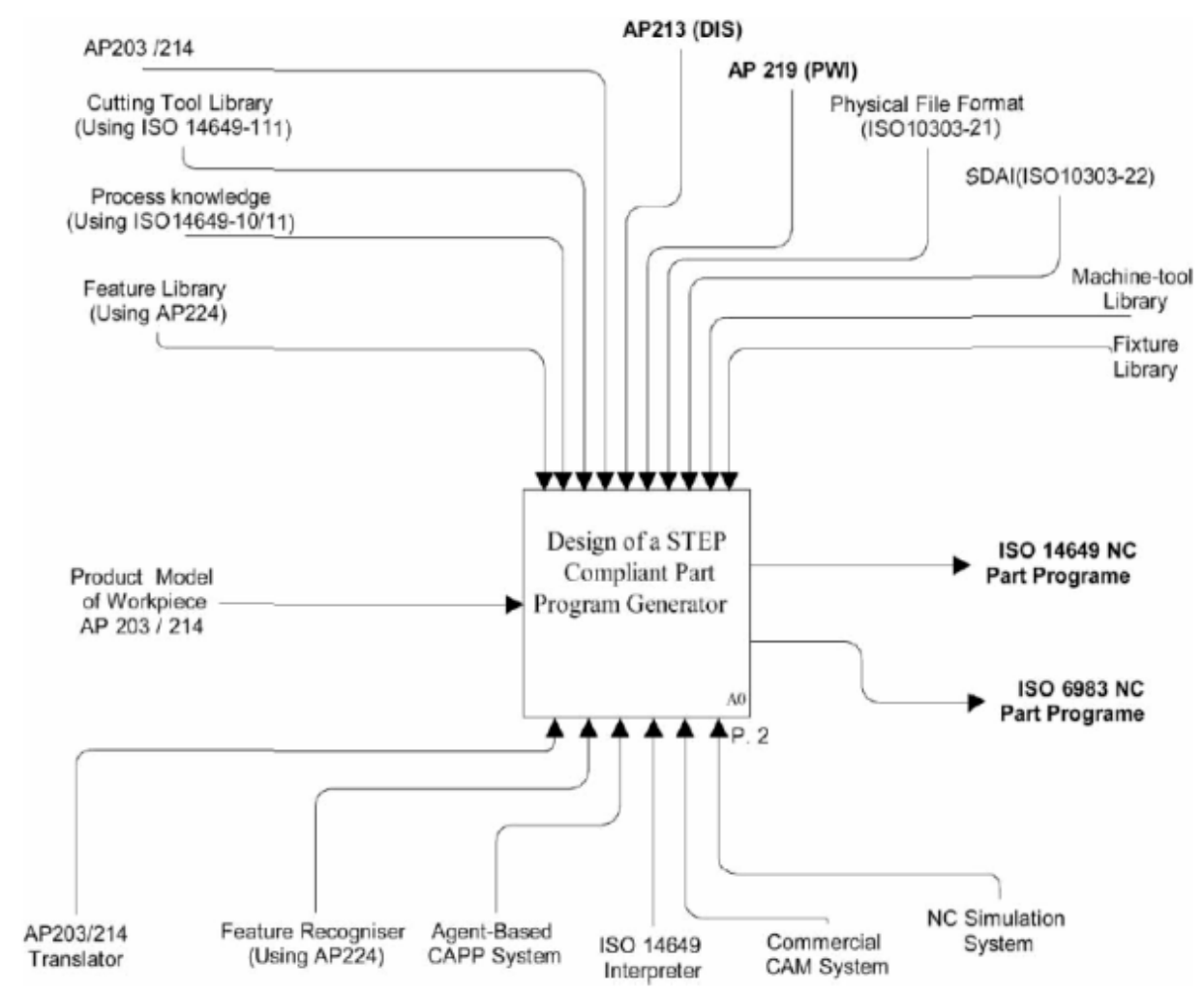

Fig. (6). Architecture of the AB-CAM system (XU, et al., 2005).

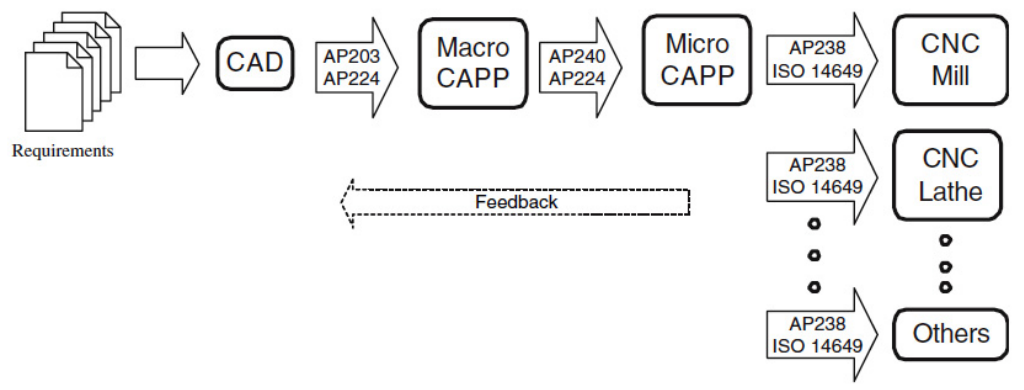

Fig. (7). Integrating design with manufacturing from (XU, et al., 2005).

product, with all the standard STEP data, and is translated into AP 224format, which defines its manufacturing information, and the process is planned. ISO AP238 and ISO14649 are the possible two results of the process planning. All the information about the product, its manufacturing requirements, processesand tool information are present in this file and allows all the safety requirements to be preprocessed before actual machining. Fig. (7) depicts how this file is utilized as an input to a CNC machine and it also illustrates the unhindered product information and data exchange and flow [2].

\subsubsection{Closing the Loop}

Once the product is manufactured it is mandatory to check the result to determine if it complies with the design parameters. A large number of tools have been designed over the years for this function such us gauge blocks, micrometres, dial indicators or more recently technologies as com- puter-controlled CMMs (Coordinate Measuring Machines) which add to the inspection process more precision and a higher speed of performance. Besides all the benefits associated with the implementation of STEP-NC in the designing and manufacturing process there is another one that has to be mentioned in this article. STEP-NC is capable of realizing the necessary inspections in the final product to ensure its quality and reliability by providing complete feedback throughout the operation chain. With this aim AP 249 has been developed to create a compliant inspection framework. A more complex and detailed database as the one given by STEP, allows easy access to the standardized product information, inspection planning information and the analysis of the acquired results. Two elements are essential for developing a closed-loop system, the "Product Information Model" and the "Manufacturing and Inspection Information model". Fig. (8) illustrates the data contained in the Product Information Model such as the geometry of the parts, material characteristics, tools which are going to be used and tolerances. 
Sources of standard information

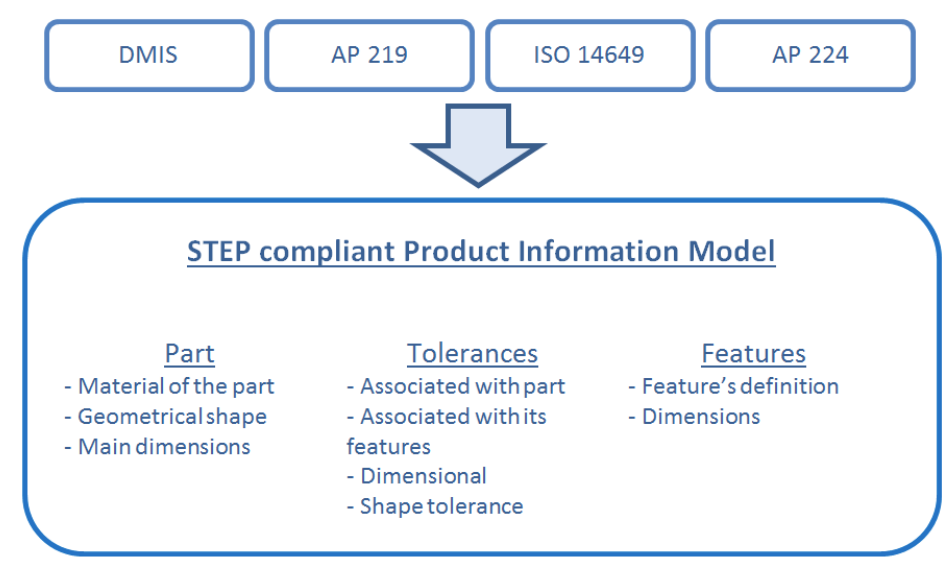

Fig. (8). Product information model for the STEP-compliant. Adapted from (Ali, et al., 2005).

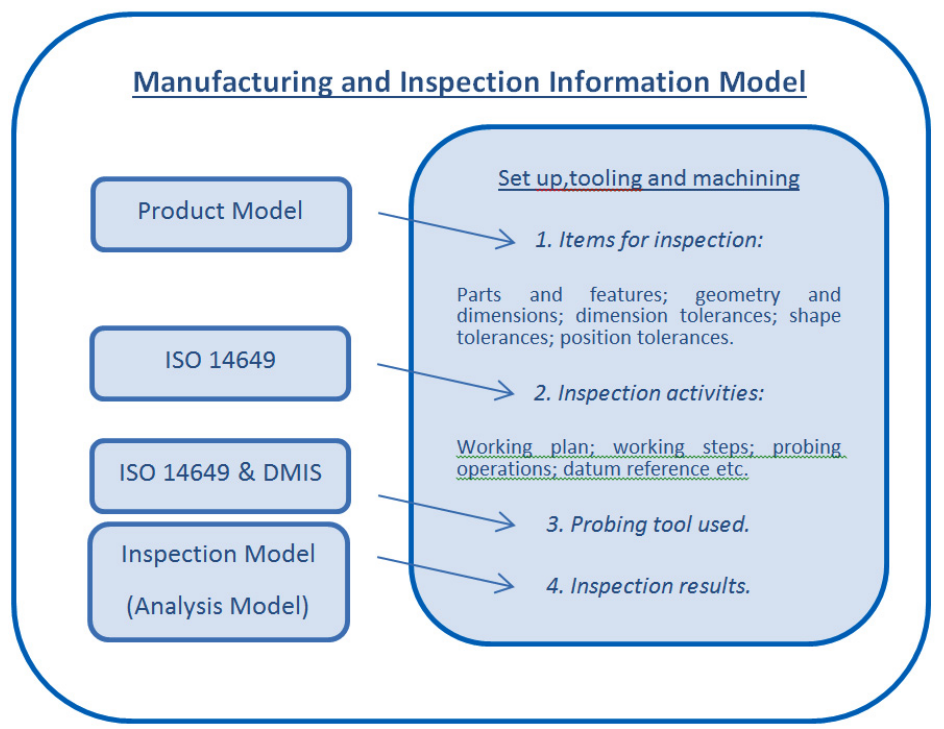

Fig. (9). Manufacturing information model for the STEP-compliant inspection framework. Adapted from (Ali, et al., 2005).

This information enables STEP-NC to create the Manufacturing/Inspection Information model (Fig. 9) which stores the inspection activities and information of the items for inspection. The result of the combination of those Information Models isa detailed procedure for the performance of the inspection which contains the inspection strategy, resources required and features involved in the operation. This inspection file can be interpreted by a CMM and eventually become part of the STEP information database making it valuable for future CAx processes [11].

\section{DISCUSSION}

\subsection{Obstacles in Implementing STEP-NC}

Throughout the research into in STEP-NC we have noticed a remarkable source of possibilities in the implementation of this system. The benefits that would be provided cover the scope of the whole product life cycle, from the design stage up to the inspection of the final product can be controlled through STEP-NC. Moreover, the disassembling at the end of the product life is able to be managed in the same way. One of the most valuable characteristic of STEP and STEP-NC is the interchangeable source of information that permits sharing all this knowledge acquired along the different projects developed with this tool and becoming part of an inestimabledatabase, and reused in future ones [12].

However, according to $\mathrm{Xu}$ and $\mathrm{He}$ (2004) the implementation of an STEP-NC system has not been developed enough to ensure notable profitability, which is the aim of any company. There are still fields related to the industry in which STEP-NC protocols have not been developed sufficiently. Therefore, implementing this $\mathrm{NC}$ protocol would be hard, increasing the probability of rejection. As in any sector of the industry, the implementation of a new system to replace the ones which have been working with a reputable profitability is a very daunting task and is not readily accepted by the CAD/CAM system community. The decision of upgrading to STEP-NC, besides the fact that it could enhance the machining process, is not only the decision of the 
designing or manufacturing department of each company, but a financial one as well in which the possible benefits provided from this change would have to be evaluated.

Analysing the benefits of STEP-NC we remarked the ability of creating a reliable database which not only contains all the information about the whole life cycle of the product but also the valuable experiences from previous machining operations. However, due to the fact that this is a quite recent standard this database is not fully formed making its development an important duty for those who opt for being pioneers in the implementation of STEP-NC. Some of the STEP-NC protocols and STEP standards are still confusing containing incomplete and inconsistent information. Moreover, the NC machining device would have to be modified and upgraded to enable them to use this information which puts an extra burden on the capital investment [2].

Furthermore, STEP-NC has been developed with the aim of being entirely portable allowing any company to physically separate the departments around the globe. There are risks involved in such a strategy such us exposing the design data and STEP-NC file information vulnerable to possible exploits. Therefore, advanced security sharing procedures would have to be implemented for transferring files between the designers, manufacturers, consultants and clients involved [3]

\subsection{Opportunities Presented by STEP-NC}

In spite of all the progress in the fields of design and manufacturing and the development of the tools used in these fields, the problems of integration and the seamless transfer of information between the different software specific and machine specific CAD, CAM and CAPP systems have always persisted. The development of STEP-NC is a major step towards rectifying this problem bystandardizing product models, methods and protocols to move towards a more globally integrated designing and manufacturing environment [8]. STEP-NC facilitates the ease of program portability among different machines and provides the feature and object oriented manufacturing procedure focusing on the machine operations performed on the part rather than the GCode machine tool axis movements to maintain repeatable quality and reliable standards [6].

The Implementation of "STandard for the Exchange of Product" (STEP) has the potential to save time and resources by decreasing lead times in manufacturing and refining the process control of the manufacturing. STEP and its offshoot STEP-NC, for machining, aim to provide a common, but a more robust and standardized database, to improve productivity, interoperability and reliability. This system compliments well with concurrent engineering methodology by standardizing machining, product feature and manufacturing procedures. Time and resources are saved by having mobile manufacturing sites or outsourcing the manufacturing altogether with the satisfaction that machine dependence, noninteroperability, non-reliability and lack of quality would not be an issue [13].

As the accuracy and repeatability is paramount for quality so is the need to regulate the manufacturing process control by reducing manufacturing mistakes and achieving very low tolerances. STEP-NC eliminates the dependence on traditional machining processes which used to rely on expertize and understandingof the machine operator and information contained in machining data books for the process parameters for manufacturing instead it provides standardized product information, right through from the design stage, independent of machine and software configurations in effect providing interoperability and seamless data flow. Having an optimized process control and utilizing the on-board inspection sensors variables such as the tool condition, temperature changes, dimensional errors and human fallibility are mitigated [12].

\section{CONCLUSION}

This paper was developed with the objective of exposing the main characteristics of a new CNC system based in the STEP standard which replaces the ISO G-Code standard which is proving to be a bottleneck for the manufacturing process. Numerous sources has been consulted to get in depth information about STEP-NC and to identify the current state of this technology. Hence, based on the information gathered, the writers of this article believe that despite some current disadvantages as consequence of STEP-NC is not being fully developed, itis the most adequate approach and due to the high levelof compatibility of the CAx systems with the manufacturing shop floor, in a short period of time wouldit may become the main standard for NC machining across the world.

\section{CONFLICT OF INTEREST}

The authors confirm that this article content has no conflict of interest.

\section{ACKNOWLEDGEMENTS}

Declared none.

\section{REFERENCES}

[1] M. Rauch, R. Laguionie, J. Y. Ha scoet, and S. H. Suh, "An advanced STEP-NC controller for intelligent machining processes," Robotics and Computer-Integrated Manufacturing, vol.28, no.3, pp. 375-384, 2012

[2] X. W. XU, H. Wang, J. Mao, S. T. Newman, T. R. K ramer, F. M. Proctor, and J. L. Michaloski, "STEP compliant NC research: the search for intelligent CAD/CAPP/CAM/CNC integration," International Journal of Production Research, 1 September, 43(17), p. 3703-3743, 2005.

[3] X. W. XU, and Q. He, "Striving for a total integration of CAD, CAPP, CAM and CNC," Robotics and Computer - Integrated Manufacturing, vol. 20, no.2, pp. 101-109, 2004.

[4] A. Nassehi, S. T. Newman, and R. D. Allen, "STEP-NC compliant process planning as an enabler for adaptive global manufaturing," Robotics and Computer - Integrated Manufacturing, vol. 22, no.56, pp. 456-467, 2006.

[5] SCRA, South Carolina Reasearch Authority, 2006. STEP APPLICATION HANDBOOK ISO 10303 VERSION 3. [Online] Available at: http://www.uspro.org/[Accessed 24 February 2013].

[6] J. Sääski, T. Salonen, and J.Paro, "Integration of CAD, CAM and NC with STEP-NC," s.l.: VTT Information Service, 2005.

[7] F. Ridwan, X. Xu, and G. Liu, "A framework for machining optimization based on STEP-NC," Journal of Intelligent Manufacturing, vol. 23, no.3, pp. 423-441, 2012.

[8] X. W. Xu, "Realization of STEP-NC enabled machining," Robotics and Computer-Integrated Manufacturing, vol. 22, no.2, pp. 144$153,2006$. 
[9] Y. Yusof, M. Fahrurazi, and M. Nor, "STEP-NC-Compliant Systems for the Manufacturing Environment," World Academy Of Science, Engineering And Technology, no. 25, pp. 922-928, 2009.

[10]S. Newman, R. Allen, and R. S. U. Rosso, "CAD/CAM solutions for STEP Compliant CNC Manufacture," INT. J. COMPUTER INTEGRATED MANUFACTURING, vol.16, no.7-8, pp. 590-597, 2003.

[11] L. Ali, S. T. Newman, and J.N. Petzing, "Developoment of a STEP-compliant inspection framework for discrete components,"
IMechE / Professional Engineering Publishing, vol. 219, no.7, pp. 557-563, 2005

[12] S. Kumar, A. Nassehi, S. T. Newman, R. D. Allen, and M. K. Tiwari, "Process control in $\mathrm{CNC}$ manufacturing for discrete components: A STEP-NC compliant framework," Robotic and Computer Integrated Manufacturing, vol.23, no.6, pp. 667-676, 2007.

[13] Y. Yusof, and K. Case, "Design of a STEP compliant system for turning operations," Robotics and Computer-Integrated Manufacturing, vol. 26, no.6, pp. 753-758, 2010 .

Received: May 28, 2015

Revised: July 14, 2015

Accepted: August 10, 2015

(C) Kayani and Rico; Licensee Bentham Open.

This is an open access article licensed under the terms of the (https://creativecommons.org/licenses/by/4.0/legalcode), which permits unrestricted, noncommercial use, distribution and reproduction in any medium, provided the work is properly cited. 